\title{
Efecto del uso de estrategias innovadoras en el proceso enseñanza-aprendizaje de las matemáticas en estudiantes de primer año universitario.
}

\author{
Hilmar Lorena Castro ${ }^{*}$ \\ ${ }^{1}$ Quality Leadership University - Panamá \\ *Autor para correspondencia. E-mail: hcastro@qlu.pa
}

Recibido: 29 de agosto de 2019

Aceptado: 16 de octubre de 2019

\begin{abstract}
Resumen
El propósito de esta investigación ha sido evaluar la implementación de estrategias innovadoras que faciliten el aprendizaje por parte de los estudiantes, y determinar el efecto de la aplicación de estas estrategias y técnicas en la enseñanza de las matemáticas a estudiantes de primer año en Quality Leadership University (QLU), específicamente en el curso de Algebra. El enfoque de esta investigación es principalmente cualitativo. La población de este estudio está representada por los estudiantes de QLU que toman el curso de Algebra en el período 1 de 2018, en total 55 estudiantes. A fin de recopilar la información relacionada al proceso de aprendizaje, se diseñaron dos instrumentos: un pre-test y un examen final. Una vez recogidos los datos del pre-test y del examen final se aplicaron herramientas estadísticas para describir los datos y tratar de hallar relaciones entre las pruebas. Del pre-test se pudo determinar que el $45 \%$ de los estudiantes no tienen las competencias necesarias para desarrollar satisfactoriamente un curso de algebra de nivel universitario. Sin embargo, al final del curso despues de aplicar las estrategias innovadoras de enseñanza, se obtuvo un 65\% de estudiantes aprobados, $10 \%$ mas de lo que se esperaba a priori. Mas aún de ese 45\% que no estaba preparado, el 40\% pudo aprobar el curso de manera satisfactoria. Se puede concluir que las estrategias innovadoras tienen un efecto positivo en el proceso de enseñanza aprendizaje de los estudiantes de primer año de QLU.

Palabras Clave : Innovación educativa; educación superior; curso universitario; enseñanza de las matemáticas; método de aprendizaje.

Abstract

The focus of this research is mainly qualitative. The population of this study is represented by QLU students taking the Algebra course in the period 1 of 2018, in total 55 students. In order to gather information related to the learning process, two instruments were designed: a pre-test and a final exam. Once the pre-test and final exam data were collected, statistical tools were applied to describe the data and try to find relationships between the tests. From the pre-test, it was determined that $45 \%$ of the students do not have the necessary skills to successfully develop a university-level
\end{abstract}


algebra course. However, at the end of the course, after applying innovative teaching strategies, $65 \%$ of approved students were obtained, $10 \%$ more than expected a priori. Moreover, of that $45 \%$ that was not prepared, $40 \%$ were able to pass the course satisfactorily. It can be concluded that innovative strategies have a positive effect on the teaching-learning process of QLU freshmen.

Keywords: Educational innovation; higher education; University course; mathematics teaching; learning method.

\section{Introducción}

El tema de la educación y de cómo tratar de mejorar la calidad de la educación en los países de Latinoamérica siempre es un tema de actualidad.

Desde hace un par de décadas, se viene escuchando sobre la necesidad de aplicar y utilizar técnicas variadas e innovadoras en la enseñanza de las ciencias y las materias asociadas con la tecnología e ingeniería, y principalmente en la enseñanza de las matemáticas (STEM). Alrededor del mundo se han ido manifestando las indudables ventajas pedagógicas de estas técnicas, y se han divulgado múltiples trabajos e investigaciones relacionadas al uso y efecto en los países mas avanzados. En Latinoamérica y en especial en Panamá se están abriendo las puertas a estas técnicas, a su divulgación y a su uso, para lograr una mejora significativa en el aprendizaje y entendimiento de estas materias, así como lograr el desarrollo de las capacidades de análisis que son necesarias para los futuros profesionales y líderes del mundo.

El propósito de esta investigación es implementar metodologías innovadoras que faciliten el aprendizaje por parte de los estudiantes, y determinar el efecto de la aplicación de estas técnicas en la enseñanza de las matemáticas a estudiantes de primer año en Quality Leadership University (QLU), específicamente en el curso de Algebra.

El objetivo general de este trabajo es determinar el efecto de la aplicación de estrategias innovadoras en el proceso enseñanza y aprendizaje de las matemáticas en estudiantes de primer año de universidad, específicamente de QLU.

Los objetivos específicos son:

- Determinar el nivel inicial de las habilidades necesarias en la resolución de problemas de Álgebra en los estudiantes de primer año de QLU.

- Seleccionar las estrategias innovadoras a usar con los estudiantes del curso de Algebra para el desarrollo del proceso de enseñanza y aprendizaje en el área de matemáticas.

- Analizar la efectividad de las estrategias utilizadas con los estudiantes de primer año de QLU en el aprendizaje de los contenidos del curso de Álgebra.

\subsection{Marco Teórico}

Este trabajo nace de la necesidad de realizar un estudio que permita identificar cuales son deficiencias en matemáticas de los estudiantes que ingresan a primer año de la universidad y determinar qué alternativas innovadoras pueden ayudar en el proceso de enseñanza-aprendizaje para facilitar el desarrollo académico en el área y lograr un exitoso encaminamiento hacia la obtención de un título universitario. 
En Panamá la educación ha venido escalando una serie de cambios que tienen como finalidad desarrollar las habilidades de aprendizaje de los estudiantes, para así mejorar la calidad de los futuros profesionales y llevándolos a ser cada día más competitivos, tanto dentro como fuera del país. El cuerpo académico en el área de las matemáticas presenta la necesidad constante de elevar su preparación, por ello se ofrecen periódicamente seminarios y talleres con el fin de actualizar tanto sus conocimientos, como las diversas metodologías que están innovando en el área de la enseñanza. Para lograr estos retos, se requiere aplicar nuevas estrategias educativas, con propuestas innovadoras, para desarrollar las habilidades, competencias y destrezas necesarias en la resolución de problemas que requieren de análisis matemático.

Los hechos que motivan esta investigación son principalmente:

- La falta de preparación observada en los estudiantes de primer año de universidad para afrontar un curso de alto nivel.

- La poca implementación de técnicas innovadoras en la enseñanza de las matemáticas.

- La alta tasa de deserción estudiantil en los primeros años de carrera, muchas veces por la frustración de no poder asimilar los cursos de matemáticas básicos en cualquier licenciatura o ingeniería.

Las preguntas que se tratan de responder en este estudio son:

- ¿Qué metodología se utilizan tradicionalmente en la enseñanza de los contenidos del curso de álgebra?

- ¿Qué innovaciones didácticas favorecen el aprendizaje del curso de Algebra de los estudiantes de primer año de QLU?

- ¿Qué factores dificultan el aprendizaje del contenido del curso de Algebra en los estudiantes de primer año de QLU?

Para el problema de estudio el enfoque es cualitativo, ya que está orientado a la exploración, descripción y entendimiento de un fenómeno (el aprendizaje), es un problema general y amplio, y además está dirigido a las experiencias de los participantes.

En cuanto al marco teórico y la revisión de la literatura, el enfoque nuevamente es cualitativo, ya que este marco contextualiza el fenómeno, justifica el planteamiento y la necesidad del presente estudio.

En cuanto a la recolección y análisis de los datos, el enfoque es cuantitativo, ya que se aplican unos instrumentos predeterminados, de los que se obtienen unos datos numéricos, a los que se le aplica análisis estadístico, se pueden describir tendencias, comparación de grupos o relación entre variables, y finalmente se comparan los resultados con predicciones de la misma data.

En cuanto al reporte de resultados considero que el enfoque es mixto, algunos resultados serán reflexivos y con aceptación de tendencias (enfoque cualitativo), pero otros serán objetivos, sin tendencias o no flexibles (enfoque cuantitativo).

Considerando entonces las características de estos enfoques se puede decir que la investigación tiene un enfoque principalmente cualitativo.

\subsection{Metodologías tradicionales en la enseñanza de las matemáticas}

Investigar la problemática de la enseñanza y del aprendizaje de las matemáticas es uno de los tópicos de mayor desarrollo desde hace muchos años. Robert y Speer (2001) ofrecen una amplia revisión de los diferentes estudios de esta problemátic a nivel mundial. 
En la metodología tradicional, según Salinas y Alanís (2009), el contenido matemático se presenta estructurado de manera formal y rigurosa. Esta presentación formal y rigurosa culmina con aplicaciones del contenido matemático que dejan la impresión de que son consecuencia natural del dominio de la teoría.

Esta presentación tradicional del contenido se vincule con una estrategia de enseñanza tradicional del profesor que se limita a exhibir (enseñar) la estructura, ya que presupone que así se dará el aprendizaje.

El estudiante, en consecuencia, adopta un papel pasivo durante el proceso de transmisión del contenido y su aprendizaje se evidencia mediante el dominio de esa estructura de conceptos y procedimientos rigurosamente organizados, aunque en mayor medida a través de su habilidad para resolver ejercicios rutinarios de corte algorítmico que han sido diseñados para facilitar al profesor la emisión de una evaluación.

En la metodología tradicional, el estudiante encuentra altas dificultades para entender los conceptos, y suelen etiquetar las matematícas como una materia abstracta, aburrida y dificil de aprender. Además, la aplicación de estos conceptos y procedimientos a problemas de análisis es un reto para el estudiante, el cual puede llegar a sentir frustación al no entender como se conecta la teoria con la práctica.

\subsection{Metodologías Innovadoras en la enseñanza de las matemáticas}

Las metodologías innovadoras tienen como fin potenciar las capacidades de los estudiantes para obtener los mejores resultados en el proceso del aprendizaje. Actualmente, muchos profesores de Latinoamérica nos sentimos con el compromiso de cambiar la educación tradicional a través del uso de estrategias innovadoras. Esto requiere que los profesores estén dedicados a aprender nuevas técnicas, a emprender nuevos rumbos en las aulas, a salir de su zona de confort para crear ambientes de aprendizajes innovadores, donde el estudiante sea activo, donde se generen actividades que despierten su interés en las matemáticas, así como en su aplicación en la vida diaria.

Según Felder y Silverman (1988) hay varios tipos de aprendices:

- Aprendices sensibles e intuitivos: a los estudiantes sensibles les gustan los hechos, datos y experimentación; los intuitivos prefieren los principios y teorías. A los sensibles les gusta resolver problemas por métodos estándar y les disgustan las "sorpresas"; a los intuitivos les gusta la innovación y les disgusta la repetición. Los sensibles son pacientes con los detalles, pero lo hacen no les gusta las complicaciones; los intuitivos se aburren con los detalles y les gusta las complicaciones. Los sensibles son buenos en memorizar hechos; los intuitivos son buenos en captar nuevos conceptos.

- Aprendices visuales y auditivos: los aprendices visuales recuerdan mejor qué ven: fotos, diagramas, flujo cuadros, líneas de tiempo, películas, demostraciones. Si algo es simplemente dicho en una clase probablemente olvidarán eso. Los aprendices auditivos recuerdan mucho de lo que escuchan y más de lo que ellos oyen y luego dicen.

- Aprendices inductivos y deductivos: los estudiantes inductivos necesitan motivación para aprender. La inducción es el estilo de aprendizaje natural del ser humano. Por otro lado, la deducción es el estilo de enseñanza humana natural, al menos para materias técnicas en la universidad. 
- Aprendices activos y reflexivos: los estudiantes activos no aprenden mucho en situaciones que requieren que ellos sean pasivos, los aprendices reflexivos no aprenden mucho en situaciones que no brindan oportunidad de pensar sobre la información que se presenta.

- Aprendices secuenciales y globales: algunos estudiantes se sienten cómodos con la presentación del material en una progresión lógica y ordenada; aprenden de forma secuencial a dominar el material más o menos como es presentado. Los estudiantes globales deben tener la libertad de idear sus propios métodos para resolver problemas en lugar de ser obligados a adoptar la estrategia del profesor, y ellos deberían estar expuestos

periódicamente a conceptos adelantados en vez de esperar a que estos conceptos sean normalmente presentados.

Las técnicas de enseñanza tradicionales, dónde el profesor es solo un expositor de los conocimientos, sólo son efectivas en algunos tipos de estudiantes, pero no llegan a todos. Por ello se reflejan altos niveles de frustración e incluso deserción en estudiantes a nivel universitario.

Las técnicas de enseñanza innovadoras son aquellas que introducen actividades en clase o a través del uso de la tecnología.

Según Felder y Silverman (1988), las características de las técnicas de enseñanza - aprendizaje de materias de ingeniería que abarcan a todos los estilos de aprendices deben ser:

- Motivar el aprendizaje. En la medida de lo posible, hay que relacionar el material que se está presentado a lo que ha venido antes y lo que está por venir el mismo curso, al material en otros cursos, y particularmente a la experiencia personal de los estudiantes (inductivo/global).

- Proporcionar un equilibrio entre la información concreta (hechos, datos, reales o experimentos hipotéticos y sus resultados) (sensibles) y el resumen de conceptos (principios, teorías, modelos matemáticos) (intuitivo).

- Equilibrar el material que enfatiza la resolución de problemas prácticos (deductivo/activo) con material que enfatiza la comprensión fundamental (intuitivo/reflexivo).

- Proporcionar ilustraciones explícitas de patrones intuitivos (inferencia lógica, reconocimiento de patrones, generalización) y detección patrones (observación de los alrededores, experimentación empírica, atención al detalle), y alentar a todos los estudiantes a ejercitar ambos patrones (sensible/intuitivo). No esperar que ninguno de los grupos pueda para ejercer los procesos del otro grupo de inmediato.

- Siga el método científico al presentar material teórico. Proporcionar ejemplos concretos de los fenómenos que la teoría describe o predice (sensible/inductivo); luego desarrollar la teoría o formular el modelo (intuitivo / inductivo / secuencial); mostrar cómo la teoría puede ser validada y deducir sus consecuencias (deductivo / secuencial); y presentar aplicaciones actuales (sensible / deductivo / secuencial).

- Usar imágenes, esquemas, gráficos y bocetos simples de forma generosa antes, durante y después de la presentación del material verbal (sensible/visual). Mostrar películas (sensible/visual). Proporcionar demostraciones (sensible/visual), práctica, si es posible (activo).

- Usar instrucción asistida por computadora: los aprendices sensibles responden muy bien a ella (sensible/ activo). 
- No llenar cada minuto de clase, disertando y escribiendo en el tablero. Proporcionar intervalos, aunque sean breves, para que los estudiantes piensen sobre lo que les han dicho (reflexivo).

- Proporcionar oportunidades para que los estudiantes hagan algo activo además de transcribir notas. Actividades de intercambio de ideas en grupos pequeños que toman no más de cinco minutos son extremadamente efectivos para estos propósitos (activo).

- Asignar algunos ejercicios de simulacro para proporcionar práctica de los métodos básicos que se enseñan (sensible / activo / secuencial) pero no exagerarlos (intuitivo / reflexivo / global). También proporcionar algunos problemas abiertos y ejercicios que requieran análisis y síntesis (intuitivo / reflexivo / global).

- Brindar a los estudiantes la opción de cooperar en las tareas asignadas en la mayor medida posible (activo). Los aprendices activos en general aprenden mejor cuando interactúan con otros; si se les niega la oportunidad de hacerlo, se les está privando de su mayor herramienta de aprendizaje efectiva.

- Aplauda las soluciones creativas, incluso las incorrectas (intuitivo / global).

El curso de Algebra de Quality Leadership Univerity es un curso cuyos objetivos son aprender el manejo de las funciones lineales, cuadráticas, polinimiales, racionales, exponenciales y logarítmicas, y la resolución de ecuaciones y desigualdades que involucren dichas funciones. Además, se estudian los dominios, rangos y operaciones con dichas funciones. El curso tiene una duración de 1 cuatrimestre, con clases presenciales, dos sesiones de 2 horas cada una (4 horas por semana). El curso es obligatorio para todas las carreras ofrecidas en Quality Leadership University.

Los cursos tienen alrededor de 22 a 25 estudiantes por grupo y se utilizaron los 3 grupos del primer cuatrimestre del 2018. Dado que la universidad posee acceso a internet tanto para estudiantes como para el profesor, y hay proyectores en cada salón, el uso de una plataforma educativa permite una comunicación bidireccional con los estudiantes, junto con el uso de herramientas tecnológicas para evaluar de forma rápida y concreta las definiciones a medida que se van estudiando.

Se implementaron metodologías innovadoras de corte tecnológico y audiovisual además de técnicas abiertas y participativas de comprensión en clases, tomadas del Proyecto Educativo en STEM realizado en Panamá (2016). Las técnicas innovadoras utilizadas en este estudio se pueden describir como:

Uso de plataforma educativa Schoology, en la cual se les publicó la información y contenido del curso, para cada capítulo se les asignó un conjunto de ejercicios que estaban disponibles allí, además se les publicaron videos explicativos de aquellas técnicas utilizadas en clase que no eran simples de entender. Cada semana se publicaban ejercicios cortos para realizar de forma remota e individual desde su casa u oficina con el objetivo de ir haciendo simulacros de los exámenes. Estas evaluaciones eran sumativas.

- Cada clase tenía un material audiovisual relativo al tópico que se estaba tratando, que les permitía relacionar la teoría con la práctica.

- Se realizaban evaluaciones de comprensión de definiciones a través de clickers virtuales (jse usó la plataforma en línea Kahoot!), algunas de ellas formativas y algunas sumativas, donde se les colocaba una pregunta y tenían un tiempo entre 30 segundos a 2 minutos para escoger 
la respuesta que consideraran correcta entre las cuatro opciones presentadas, obteniendo al final porcentajes de respuestas correctas así como la retroalimentación de cada una de las preguntas.

- Cada semana se realizaban clases solo de práctica, formativas, donde los estudiantes tenían la oportunidad de trabajar en grupos e individual la solución de los ejercicios asignados para esa semana, junto con el apoyo del profesor del curso.

- En las clases presenciales se les impartía los nuevos tópicos, tratando de relacionar el contenido previo con el contenido de la clase y haciendo énfasis en el uso a futuro de esos contenidos.

\section{Metodología}

Las fuentes de información utilizadas para la elaboración de esta investigación son principalmente artículos obtenidos a través de las librerías virtuales, así como trabajos de grado relacionados con el problema de investigación. Además, se utilizaron los informes de la Unesco de Educación Para Todos de los últimos dos años para Latinoamérica y artículos extraídos de internet que tenían relevancia con el tema.

Del universo de estudiantes de QLU, solo se tomaron estudiantes que estuvieran cursando el curso de Algebra en el período 1 del año 2018. La muestra final excluye estudiantes mayores de 35 años (sólo había un estudiante mayor de 35 años, que estaba estudiando su segunda carrera), ya que no coinciden con el perfil de los estudiantes tradicionales de primer año de universidad, para un total de 65 estudiantes. Sin embargo, el estudio se realizo al final con 55 estudiantes, ya que 10 de los 65 estudiantes originales abandonaron el curso.

De la hipótesis planteada se pueden extraer las siguientes variables:

- Variable Independiente: estrategias innovadoras en la metodología de la enseñanzaaprendizaje en el curso de Algebra

- Variable Dependiente: aprendizaje de los estudiantes.

Para la realización de este estudio se crearon dos instrumentos, ambos son evaluaciones de contenido, uno es un pretest y otro es un posttest.

\subsection{Descripción de los Instrumentos}

El éxito de un investigación depende en buena parte de la pertinencia de las técnicas seleccionadas para la recolección de la información, así como la calidad de los instrumentos utilizados para tal fin. Según Arias (2006), la técnica de recolección representa el conjunto de procedimientos o foemas utilizadas en la obtención de la información necesaria para lograr los objetivos de la investigación. En la presente investigación se utilizaron dos instrumentos: un pre-test y un examen final. El pre-test es una versión del test de College Board llamado ACCUPLACER, cuyo propósito es proporcionar información útil sobre sus habilidades académicas en matemáticas, lectura y escritura de un individuo. Más de 8 millones de pruebas son tomados cada año a nivel mundial. Es una prueba que le ayuda a identificar fortalezas y necesidades en cada área temática. 
La prueba consiste en 30 preguntas de selección múltiple, con una única respuesta correcta por pregunta, y 60 minutos para responder. Las preguntas incluyen los temas básicos de números enteros y racionales, expresiones algebraicas, ecuaciones lineales, desigualdades lineales, operaciones algebraicas, ecuación de la línea y aplicaciones. Para considerar que el nivel es básico se usó la misma escala que el test de accuplacer usa, es decir más de $53.33 \%$ de respuestas correctas, que corresponde a 16 respuestas correctas o más.

Accuplacer es una prueba que tiene un nivel de confiabilidad de 0.90 y está validado como prueba para dar información sobre las habilidades de un individuo en matemáticas, según Mattern y Packman (2009).

El segundo instrumento utilizado es una prueba final, que consta de 12 preguntas, algunas con varios ítems, con un tiempo para culminarla de 2 horas y realizada después de aplicar las técnicas innovadoras a los estudiantes del curso, la cual tiene un nivel de confiabilidad de 0.89 y es una prueba validada por expertos en el área.

\subsection{Recolección de la información}

Para el primer instrumento, el pre-test, fue utilizado el primer día de clases del semestre 1 del 2018, en la clase de Algebra.

El post-test fue aplicado al final del curso, tres meses después del pre-tes.

Ambas pruebas administradas se encuentran en físico en las instalaciones de QLU, en un archivo para su futura referencia o uso, y se encuantran anexadas al final del presente informe.

\subsection{Análisis de los datos}

Una vez recogidos los datos del pre-test se procedió a su procesamiento y tabulación. Los resultados de este instrumento se analizaron mediante estadística descriptiva, utilizando medidas de tendencia central (media y mediana) y de dispersión (coeficiente de variación y desviación estandár), tablas de frecuencia, y gráficas para describir los datos obtenidos.

Los resultados de la prueba final y de la nota final obtenida por cada estudiante se procesó de manera similar utilizando el paquete estadístico $\mathrm{R}$ para realizar las estadísticas descriptivas, asi como el análisis de un modelo de regresión y correlación entre las pruebas.

\section{Resultados y Discusión}

El pre-test fue tomado de las 3 secciones (Grupo 1, Grupo 2 y Grupo 3) de Algebra en el semestre 1 de 2018 en QLU.

\section{Cuadro 1: Resultados del Pre-test}

\begin{tabular}{|c|c|c|c|c|}
\hline Respuestas correctas & Grupo 1 & Grupo 2 & Grupo 3 & Total \\
\hline 0 a 7 & 0 & 1 & 3 & 4 \\
\hline 9 a 15 & 7 & 6 & 8 & 21 \\
\hline 16 a 22 & 7 & 6 & 4 & 17 \\
\hline 23 a 30 & 4 & 6 & 3 & 13 \\
\hline Total & 18 & 19 & 18 & 55 \\
\hline
\end{tabular}


Cuadro 2: Promedios del Pre-test por grupos

\begin{tabular}{|l|c|c|c|}
\hline Grupo & Total & Promedio & Desviación estandard \\
\hline Grupo 1 & 18 & 18.8 & 4.98 \\
\hline Grupo 2 & 19 & 18.2 & 6.90 \\
\hline Grupo 3 & 18 & 14.9 & 5.47 \\
\hline
\end{tabular}

Se realizaron pruebas estadísticas de homogeneidad de varianza y de promedios entre los grupos, obteniendose que en prueba Bartlett de Homogeneidad de Varianza el p-value es 0.7588, que no es menor que el nivel de significancia escogido $95 \%(\alpha=0.05)$, por lo que no hay evidencia estadística de que las varianzas entre los grupos sean diferentes. Para los promedios, el p-value obtenido de la tabla ANOVA es de 0.116, el cual no es menor que el nivel de significancia escogido 95\% ( $\alpha=$ 0.05), por lo que no hay evidencia estadística de que los promedios entre los grupos sean diferentes. Por eso se puede trabajar con la muestra general como un solo grupo, y se tiene que 30 de 55 estudiantes alcanzaron un puntaje superior a los 16 de 30 puntos en el pre-test, es decir un 55\% de los estudiantes estan por encima del nivel básico requerido para un curso de Algebra de nivel universitario.

Cuadro 3: Porcentajes de Estudiantes Preparados para un curso universitario de Algebra

\section{Resultados del Pretest}

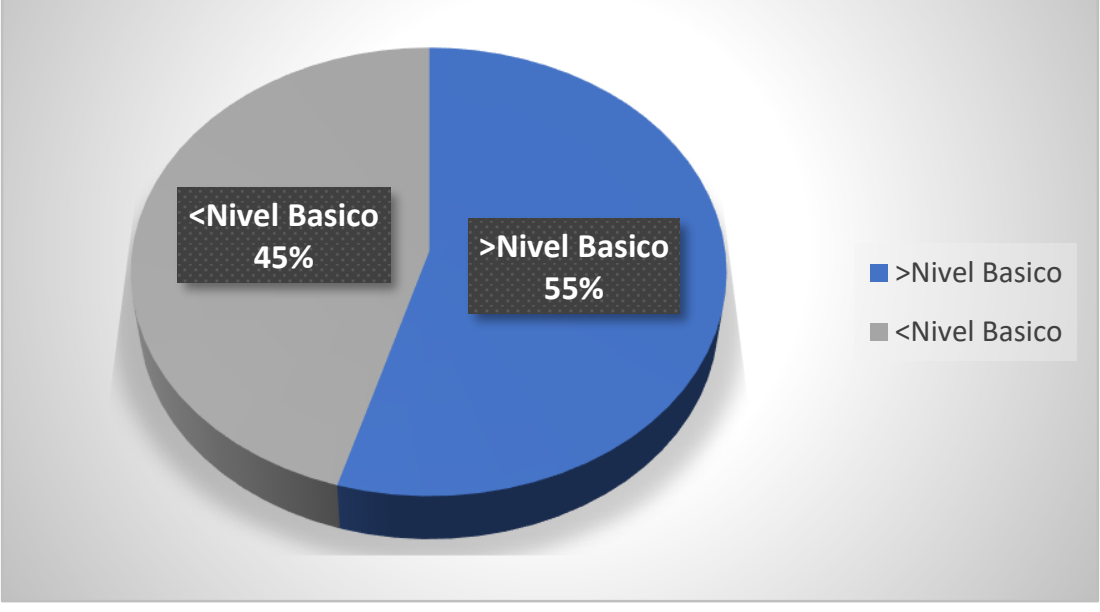

\begin{tabular}{|c|c|}
\hline $\begin{array}{l}\text { Nota del Examen } \\
\text { Final }\end{array}$ & Frecuencia \\
\hline $0-59$ & 14 \\
\hline $60-69$ & 9 \\
\hline $70-79$ & 11 \\
\hline $80-89$ & 5 \\
\hline $90-100$ & 16 \\
\hline
\end{tabular}

Del cuadro 4 se puede observar que le examen final fue aprobado (obtuvo mas de 70) por 32 de los 55 estudiantes de la población, eso corresponde a 58.2\% de los estudiantes. 
En cuanto a las notas finales del curso, se obtuvieron los siguientes resultados:

Cuadro 5: Resultados Finales del Curso

\begin{tabular}{|l|c|}
$\begin{array}{l}\text { Nota } \\
\text { Final }\end{array}$ & Frecuencia \\
\hline A & 13 \\
\hline A- & 2 \\
\hline B+ & 2 \\
\hline B & 1 \\
\hline B- & 1 \\
\hline C+ & 6 \\
\hline C & 11 \\
\hline C- & 0 \\
\hline D+ & 4 \\
\hline D & 5 \\
\hline D- & 0 \\
\hline F & 10 \\
\hline
\end{tabular}

El curso se considera aprobado si la nota final es C o mayor a C. Para este estudio 36 de 55 estudiantes $(65.5 \%)$ obtuvieron una nota aprobatoria según el cuadro 5.

Cuadro 6: Notas del Post-test vs el Pre-test

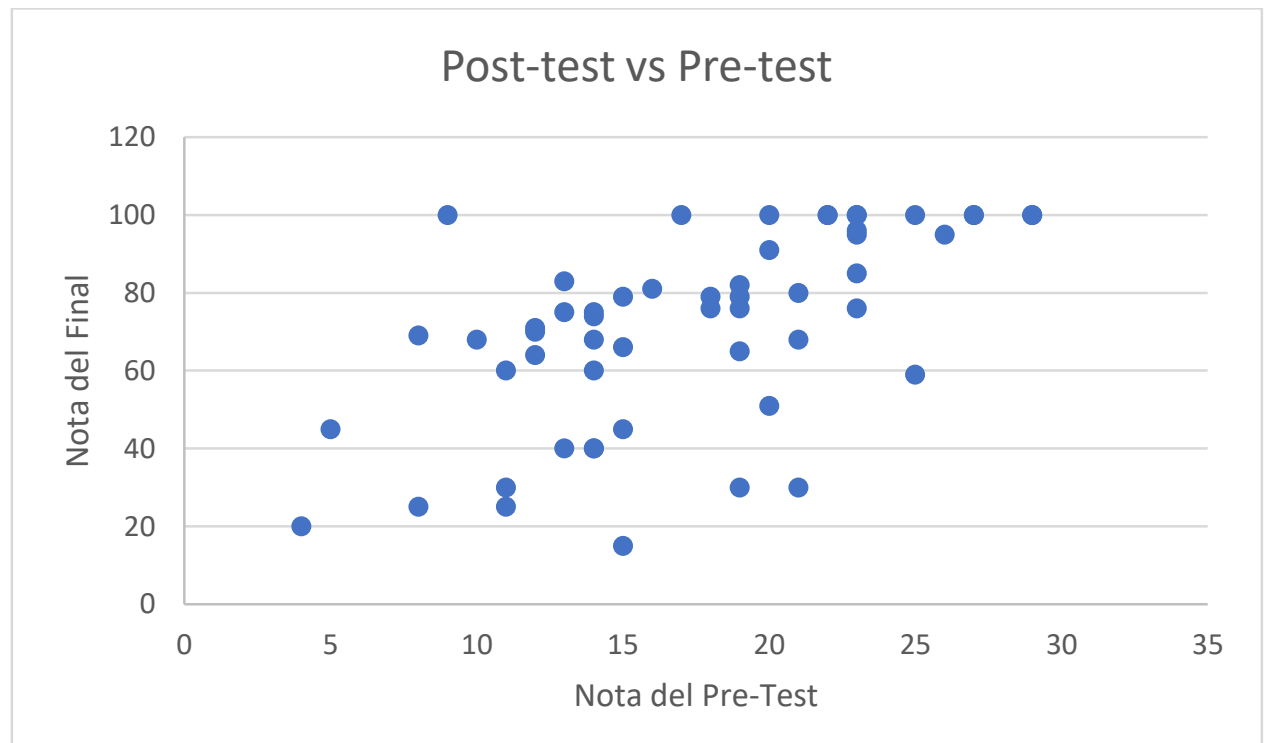

La nota final del examen no tiene una relación lineal con la nota del pre-test, hay estudiantes con una nota en el pre-test baja y con una nota alta en el pre-test. Se hizo una regresión lineal entre la nota del pre-test y la nota final del curso, donde se obtuco un $\mathrm{R}^{2}$ de 0.359 , que significa que la nota final del curso solo es explicada por la nota del pre-test en un $35.9 \%$, es decir qe hay un $64.1 \%$ de la nota final que no puede ser descrita por la nota del pre-test.

Ese porcentaje de la nota que no puede ser explicado por los resultados del pre-test, se explica por las metodologías aplicadas en el curso en sí. Después de utilizar las metodologías no tradicionales en 
este curso, se puede observar que hay personas que a pesar de que su pre-test fue muy bajo, su nota final fue excelente. Además, después del uso de estas metodologías hubo un incremento en el porcentaje de aprobados de 55\% a 65\%, lo que indica que las estrategias tuvieron un efecto positivo en la población estudiantil que tomó el curso.

\begin{tabular}{|l|c|c|c|}
\hline \multicolumn{3}{|l|}{ Cuadro 7: Estudiantes Aprobados Vs Nota del Pre-test } \\
\hline Nota & Reprobaron & Aprobaron & Total \\
\hline $\mathbf{0 - 1 5}$ & 15 & 10 & 25 \\
\hline $\mathbf{1 6 - 1 3}$ & 4 & 26 & 30 \\
\hline 7:Total & 19 & 36 & 55 \\
\hline
\end{tabular}

Del cuadro 7 se puede observar que de los 25 estudiantes que no tenian una nota en el pre-test satisfactoria, 10 de esos 25 estudiantes (40\%) aprobó satisfactoriamente el curso.

\section{Conclusiones}

Se puede concluir que la aplicación de estrategias innovadoras en el proceso enseñanza - aprendizaje de las matemáticas en estudiantes de primer año de universidad, específicamente de QLU, tuvo un efecto positivo, ya que se incrementó el porcentaje de estudiantes que a priori, por los resultados del pre-test, se esperaba que aprobaran.

Se determinó que inicialmente, un poco mas de la mitad del curso (55\%) tenia las habilidades mínimas necesarias para completar un curso universitario de Algebra satisfactoriamente.

De los estudiantes que no tenían el nivel mínimo para aprobar el curso, un 40\% de ellos obtuvieron resultados satisfactorios después de ser expuestos a las metodologías innovadoras en la clase.

Las metodologías innovadoras que se orientan para llegar a todos los tipos de estudiantes que tenemos, tienen resultados satisfactorios tanto para los estudiantes como para los profesores que se comprometen en mejorar la calidad educativa de Latinoamérica y el mundo.

\section{Bibliografía}

Arias, F. G. (2006). Mitos y errores en la elaboración de Tesis y Proyectos de Investigación. 3ra. Fidias G. Arias Odón.

Felder, R. M., \& Silverman, L. K. (1988). Learning and teaching styles in engineering education. Engineering education, 78(7), 674-681.

Mattern, K. D., \& Packman, S. (2009). Predictive Validity of ACCUPLACER ${ }^{\circledR}$ Scores for Course Placement: A Meta-Analysis. Research Report No. 2009-2. College Board.

Robert, A. \& Speer, N. (2001). Research on the teaching and learning of Calculus/Elementary analysis. In D. Holton (Ed.), The teaching and learning of mathematics at university level: An ICMI study (pp. 283-299). Holland: Kluwer Academic. 
Salinas, Patricia, \& Alanís, Juan Antonio. (2009). Hacia un nuevo paradigma en la enseñanza del Cálculo dentro de una institución educativa. Revista latinoamericana de investigación en matemática educativa, 12(3), 355-382.

UNESCO (2015), La Educación Para Todos, 2000-2015: Logros Y Desafíos, Recuperado de http://unesdoc.unesco.org/images/0023/002325/232565s.pdf

UNESCO (2016), Texto 1: Innovación Educativa, Serie "Herramientas de apoyo para el trabajo docente", Perú, Recuperado de http://unesdoc.unesco.org/images/0024/002470/247005s.pdf 


\section{DIAGNOSTIC TEST}

INSTRUCTIONS

Answer all question.

Calculator are not allowed.

Time : 60 minutes

\section{ANSWER SHEET}
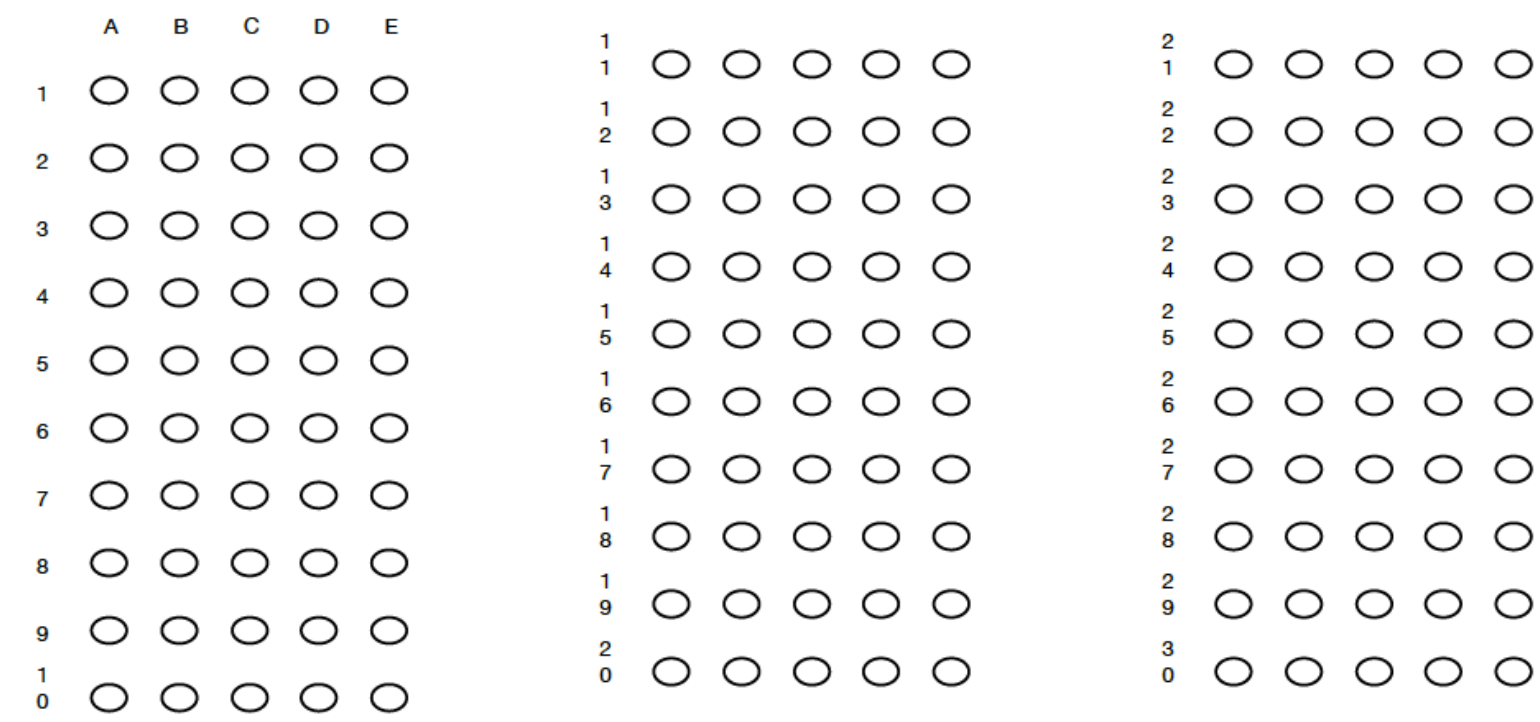

SCORE : 
1. $54-6 \div 2+6=?$
A. 6
B. 24
C. 27
D. 30
E. 57

2. Simplify: $20+4^{3} \div(-8)$
A. 4
B. -4
C. 12
D. -10.5
E. -3.5

3. The lowest temperature on a winter morning was $-8^{\circ} \mathrm{F}$. Later that same day the temperature reached a high of $24^{\circ} \mathrm{F}$. By how many degrees Fahrenheit did the temperature increase?
A. $3^{\circ}$
B. $8^{\circ}$
C. $16^{\circ}$
D. $24^{\circ}$
E. $32^{\circ}$

4. If $\left(\frac{3}{4}-\frac{2}{3}\right)+\left(\frac{1}{2}-\frac{1}{3}\right)$ is calculated and the answer reduced to simplest terms, what is the denominator of the resulting fraction?
A. 24
B. 12
C. 6
D. 4
E. 3

5. $\frac{1}{2}+\left(\frac{2}{3} \div \frac{3}{4}\right)-\left(\frac{4}{5} \times \frac{5}{6}\right)=$ ?
A. $\frac{1}{16}$
B. $\frac{17}{27}$
C. $\frac{13}{18}$
D. $\frac{7}{9}$
E. $\frac{5}{6}$

6. In scientific notation, $20000+3400000=$ ?
A. $3.42 \times 10^{6}$
B. $3.60 \times 10$
C. $3.42 \times 10^{7}$
D. $3.60 \times 10^{7}$
E. $3.60 \times 10^{12}$

7. Express in scientific notation: 0.0000056
A. $5.6 \times 10^{-6}$
B. $5.6 \times 10^{6}$
C. $5.6 \times 10^{-5}$
D. $5.6 \times 10^{-7}$
E. $5.6 \times 10^{5}$

8. Saying that $4<\sqrt{x}<9$ is equivalent to saying what about $x$ ?
A. $0<\mathrm{x}<5$
B. $0<x<65$
C. $2<x<3$
D. $4<x<9$
E. $16<x<81$

9. Simplify: $(2 a-4)+2(a-5)-3(a+1)$
A. $7 a-11$
B. $a-17$
C. $a-11$
D. $7 \mathrm{a}-17$
E. $-a-11$

10. Expand: $1.20 \times 10^{5}$
A. 12000000
B. 1200000
C. 120000
D. 12000
E. 1200 
11.What value of $x$ solves the following proportion?
A. $5 \frac{1}{3}$
B. $6 \frac{3}{4}$
C. $10 \frac{1}{2}$
$\frac{9}{6}=\frac{x}{8}$

12. Solve: $8(x-2)-5(x+4)=20+x$
A. $x=9$
B. $x=28$
C. $x=-8$
D. $x=-18$
E. $x=16$

13. On a math test, 12 students earned an A. This number is exactly $25 \%$ of the total number of students in the class. How many students are in the class?
A. 15
B. 16
C. 21
D. 30
E. 48

14. If $x=-3$, what is the value of $\frac{x^{2}-1}{x+1}$
A. -4
B. -2
C. 2
D. $3 \frac{2}{3}$
E. 5

15. Which of the following is equivalent to $3 a+4 b-(-6 a-3 b)$ ?
A. $16 a b$
B. $-3 a+b$
C. $-3 a+7 b$
D. $9 a+b$
E. $9 a+7 b$

16. Firefighters use the formula $S=0.5 P+26$ to compute the horizontal range $S$ in feet of water from a particular hose, where $\mathrm{P}$ is the nozzle pressure in pounds. Find the horizontal range if pressure is $90 \mathrm{lb}$.
A. 44 feet
B. 450 feet
C. 19 feet
D. 71 feet
E. 55 feet

17. Simplify: $2 x^{2}\left(-3 x^{2}\right)^{3}$
A. $54 x^{12}$
B. $18 x^{8}$
C. $-18 x^{12}$
D. $-54 x^{8}$
E. $18 x^{12}$

18. Simplify $\left(\frac{2 u^{-5} v^{2}}{8 w}\right)^{-2}$
A. $\frac{w^{2} v^{4}}{4 u^{7}}$
B. $\frac{16 v^{4}}{w^{2} u^{10}}$
C. $\frac{16 w^{2} u^{10}}{v^{4}}$
D. $\frac{u^{7} v^{4}}{4 w^{2}}$
E. $\frac{4 u^{7} v^{4}}{w^{2}}$

19. What is the sum of the polynomials $3 a^{2} b+2 a^{2} b^{2}$ and $-a b^{2}+a^{2} b^{2}$ ?
A. $3 a^{2} b-a b^{2}+3 a^{2} b^{2}$
B. $3 a^{2} b-a b^{2}+2 a^{2} b^{2}$
C. $2 a^{2} b+3 a^{2} b^{2}$
D. $2 a^{2} b^{3}+2 a^{4} b^{4}$
E. $-3 a^{3} b^{3}+2 a^{4} b$ 


\section{Anexo 2: Post Test}

Name :

May 3rd, 2018

Answer all the questions.

You must show all your work. An answer, right or wrong, without the proper justification will receive little to no credit. The use of an electronic calculator is expected, where appropriate.

You are reminded of the need for clear presentation in your answers.

At the end of the examination, fasten all your work securely together.

The number of marks is given in brackets [] at the end of each question or part question.

The total number of marks for this exam is 100 points.

1. Find the domain of the following functions:
(A) $f(x)=x^{2}-16 x+3$
Domain =
(B) $f(x)=\frac{12 x+5}{x^{2}-36}$
Domain $=$
(C) $f(x)=\sqrt{x^{2}-36}$
Domain $=$
(D) $f(x)=\ln \left(x^{2}-36\right)$
Domain =
(E) $f(x)=e^{x^{2}-36}$
Domain =

2. Evaluate $f(x)$ at $x=-4, x=2$ and $x=4$. State the domain of $f(x)$.

$f(x)=\left\{\begin{array}{llr}-4 x & \text { if } & x \leq-4 \\ x^{3}+2 & \text { if }-4<x \leq 2 \\ 4-x^{2} & \text { if } & x>2\end{array}\right.$

Answer: $f(-4)=$ , $f(2)=$

$f(4)=$ Domain $=$

3. (A) Find the standard equation of the circle that has a diameter with end points $(-4,3)$ and $(6,9)$.

Answer: $f(x)=$

(B) Find a symbolic representation for $\mathrm{f}^{-1}(\mathrm{x})$. Identify the domain and range of $\mathrm{f}^{-1}$.

$$
f(x)=\frac{x-1}{2 x+1}
$$

Answer : $f^{-1}(x)=$ Domain = Range = 
(C) Find the composite function $\left({ }^{\circ} \mathrm{g}\right)(\mathrm{x})$ if $\mathrm{f}(\mathrm{x})=\frac{\mathrm{x}}{3 \mathrm{x}+2}$ and $\mathrm{g}(\mathrm{x})=\frac{2}{\mathrm{x}-3} \quad$. Identify its domain.

Answer :

4. Locate any vertical and horizontal asymptote of the following function.

$$
\begin{aligned}
f(x)=\frac{x^{2}+3 x-4}{x^{2}+5 x-6} & x \text {-intercepts } \quad y \text {-intercepts } \\
& \text { Vertical asymptotes } \\
& \text { Horizontal asymptotes }
\end{aligned}
$$

5. For the graph of $f(x)=2 x^{2}+8 x-3$,
(A) Is the point $(1,12)$ on the graph of $f$ ?
(B) Write in the vertex form $f(x)=a(x-h)^{2}+k$
(C) Identify the vertex of $f(x)$
(D) What is the domain of $f(x)$ ? What is the range of $f(x)$ ?

Answer :

Answer :

Answer :

Domain :

Range :

(E) List the $x$-intercepts, if any, of the graph of $\mathrm{f}$.

Answer :

(F) List the y-intercept, if there is one, of the graph of $f$.

Answer :

(G) Where the function is decreasing? What is the minimum value of $f(x)$ ?

Answer :

Mlnimun :

6. Find the slope-intercept form for the line satisfying the conditions

(A) Perpendicular to $3 y+5 x=12$ passing through $(-2,3)$

Answer :

(B) Parallel to $3 y+5 x=12$, passing through $(1,-3)$

Answer : 
7. Write a polynomial $f(x)$ in complete factored form that satisfies the conditions: Degree 5, leading coefficient 4 and zeros $3,-2 i$ and $-5 i$.

Answer $f(x)=$

8. Write in the complete factored form of the polynomial $f(x)=x^{4}+2 x^{3}-13 x^{2}-14 x+24$

Answer:

9. Solve the following:

[5 each]

\begin{tabular}{|l|l|}
\hline a. $\frac{5-x}{x^{2}-x-2}<0$ & Answer: \\
\hline b. $\sqrt{2 x-4}+2=\sqrt{3 x+4}$ & Answer: \\
\hline c. $3 n^{-2}-19 n^{-1}+20=0$ & Answer: \\
\hline d. $|3 t-5|+3<13$ & Answer: \\
\hline e. $2 x^{3} \leq 3 x^{2}+5 x$ & Answer: \\
\hline
\end{tabular}

10. Expand $\ln \left(\frac{5 x^{2} \sqrt{y}}{w^{4}}\right)$.

[2 points]

Answer :

11. Write the expression as a logarithm of a single expression.

[3 points]

$\log _{3} x+\log _{3} \sqrt{x+3}-\frac{1}{3} \log _{3}(x-4)$

Answer : 
12. The size $P$ of a certain insect population at time $t$ (in days) obeys the function $P(t)=250 e^{0.03 t}$.

(A) Determine the number of insects at $\mathrm{t}=0$ days.

(C) What is the population after 10 days?

(D) When will the insect population reach 700 ?

Answer $[1]$

Answer _ [2]

Answer [2]

13. Solve the following equations:
a. $\ln \left(x^{2}-1\right)-\ln (x+1)=\ln 2$
b. $7^{1-2 x}=10^{5 x}$

Answer

Answer

\section{FORMULAE}

The distance between $P_{1}=\left(x_{1}, y_{1}\right)$ and $P_{2}=\left(x_{2}, y_{2}\right)$ is

$$
d\left(P_{1}, P_{2}\right)=\sqrt{\left(x_{2}-x_{1}\right)^{2}+\left(y_{2}-y_{1}\right)^{2}}
$$

The standard form of a circle with radius $r$ and center at $(h, k)$ is:

$$
(x-h)^{2}+(y-k)^{2}=r^{2} \quad r>0
$$

$\log _{a} x=k$ is equivalent to $x=a^{k}$.

$\log _{a} a^{x}=x$

1. $\log _{a} 1=0$ and $\log _{a} a=1$

2. $\log _{a} m+\log _{a} n=\log _{a}(m n)$

3. $\log _{a} m-\log _{a} n=\log _{a}\left(\frac{m}{n}\right)$

4. $\log _{a}\left(m^{r}\right)=r \log _{a} m$ 